\title{
Rechtsgeschichte
}

http://www.rg-rechtsgeschichte.de/rg7

$\operatorname{Rg} 2005 \quad 189-190$

Zitiervorschlag: Rechtsgeschichte Rg 7 (2005)

http://dx.doi.org/10.12946/rg07/189-190

\section{Thorsten Keiser}

\section{Law after Auschwitz - Law in Auschwitz?}




\section{Law after Auschwitz - Law in Auschwitz?*}

»Law after Auschwitz«, das jüngste Buch des kanadischen Rechtswissenschaftlers David Fraser, ist keine streng durchgeführte Abhandlung mit nur einer strukturbildenden Fragestellung, sondern eine Sammlung von historischen, philosophischen und soziologischen Betrachtungen über den Holocaust, das Recht, die Justiz und die Juristen in verschiedenen Ländern. Anders als der Titel vermuten lässt, geht es Fraser keineswegs darum, die Erfahrung der Massenvernichtung als einzigartigen Zivilisationsbruch und nachhaltigste Zäsur in der Menschheitsgeschichte darzustellen. Das Buch reiht sich nicht in die inzwischen lange Serie von Studien ein, die aus einem »Davor « und »Danach«-Blickwinkel Auschwitz auf seine Folgen hin untersuchen, wie es bekanntlich für >das Denken ‘ im Allgemeinen, die Theologie, die Möglichkeit, Gedichte zu schreiben u.s.w. bereits geschehen ist. Fraser setzt der Grundrichtung dieser Literaturgattung eine drastische Kontinuitätsbehauptung entgegen: Auch bei den Normen, die den Massenmord regelten, handelte es sich um Recht und damit um ein Instrument, das heute nach wie vor ähnlich verwendet wird. Kritisiert werden Deutungsmuster, die das Recht unter der NS-Herrschaft als Nicht-Recht, Rechtsperversion oder Legalitätsfassade etikettieren und damit aus dem Rechtsgeschichtsverlauf ausklammern wollen. Auch Verbrechen können und konnten (im formellen Sinn) legal sein: Nazi law was law. Diese These ist eine Art Gravitationszentrum, das die weit in verschiedene Bereiche ausgreifende Darstellung (sie enthält Auseinandersetzungen mit der Philosophie Agambens sowie mit verschiedenen juristischen Behandlungen von NS-Verbrechen in Frankreich, den USA,
Großbritannien, Kanada und Australien) vor dem Zerfall in völlig unzusammenhängende Einzelteile bewahrt. So gerät das Buch zu einem Thema mit Variationen; kaum ein Kapitel, in dem Fraser nicht dem heutigen Juristen vor Augen führt, dass sein Stoff und seine Methoden sich in vielen Aspekten nicht wesentlich von denen seiner deutschen Kollegen aus der Zeit von I933 bis I945 unterscheiden: "... there is no inherent epistemological, ontological juridical set of ideas, concepts or practices which distinguishes nazi law from our law. " Hier hat man schon eine etwas spezifischere Variante der These vom NS-Recht als beständigem Recht, die dennoch viele Fragen offen lässt. Worin das »set of ideas «, das sich fortsetzt, genau besteht, kann sich der Leser mosaikartig aus Einzelbeispielen (etwa Berichten über Euthanasietendenzen der Rechtsprechung im heutigen Großbritannien) zusammensetzen, definiert wird dieser Grundbegriff aber nicht. Doch aus der vereinfachenden Zuspitzung bezieht die These ihren Schwung; sie wirkt provozierend und wurde so drastisch wahrscheinlich noch nicht formuliert. $\mathrm{Ob}$ sie völlig neu ist, ist hingegen eine andere Frage. Zumindest die wichtige Teilaussage von Frasers Annahme, dass der NS-Staat sich auch bei der Massenvernichtung rechtlicher Normen bediente, ist beispielsweise schon von Ernst Fraenkels zeitgenössischer Beobachtung nicht geleugnet worden. Fraenkel wollte den Maßnahmenstaat nicht als formell-rechtsfreien, d. h.: völlig normlosen Raum verstanden wissen, wie man an seinen präzisen Ausführungen zu gesetzlichen Rechtfertigungsmustern des verbrecherischen Systems erkennen kann. Es gab, Fraenkel zufolge, normgesteuerte Maßnahmen, nur war

\footnotetext{
* David Fraser, Law after

Auschwitz. Towards a Jurispru-

dence of the Holocaust, Dur-

ham, N.C.: Carolina Academic

Press 2005, XI, 45 I S.,

ISBN 0-89089-243-I
} 
ihre gerichtliche Nachprüfbarkeit beschränkt. Unter »Recht « verstand Fraenkel allerdings etwas anderes. Bei Fraser ist dagegen wörtlich die Rede vom »Nazi-Rechtsstaat «, ohne, wie Fraenkel es getan hätte, formelle und materielle Rechtsstaatsbegriffe zu unterscheiden.

In dieser Definitionsfrage des Rechtsbegriffs liegt ein zentrales Problem der Darstellung. Definiert man Recht (gegen seine metaphorische Aussage) neutral als bloßen Komplex von handlungsleitenden Satzungen, die beliebige Folgen, auch Ausgrenzung und Vernichtung, herbeiführen können, und nicht als Komplex von Regeln mit immanenter Eignung zu einer die individuelle Würde schützenden Ordnung eines Gemeinwesens, dann lässt sich leicht behaupten, dass NS-Recht Recht war. So hat man gelegentlich den Eindruck, dass Fraser zu variabel mit seinem Rechtsbegriff verfährt, um die wichtigen Fragen konsequent erörtern zu können.

Aus der Sicht strenger Rechtshistoriker könnte man das Buch und seine These in manchen Aspekten auch als vergebene Chance ansehen. Fraser beschreibt leider nicht genau, wie dieses "NS-Recht « im Hinblick auf den Holocaust im Einzelnen funktionierte, er geht nicht z. B. auf die Problematik der Schutzhaftanordnungen oder der justizfreien Hoheitsakte ein, obwohl sich gerade hier noch viele Anknüpfungspunkte geboten hätten. Überhaupt scheint die quellengestützte Aufarbeitung vergangener Situationen nicht sein Anliegen zu sein. Die historischen Passagen über NS-Deutschland sind ausschließlich der englischsprachigen Sekundärliteratur oder englischen Übersetzungen deut- scher Texte entnommen; die Darstellung steuert eher in die Breite als in die Tiefe. Die meisten einschlägigen Studien über Recht im Nationalsozialismus, die in Deutschland seit Ende der sechziger Jahre entstanden sind (z. B. von Rüthers oder Gruchmann), bleiben unberücksichtigt, was aber wohl nicht auf Geringschätzung, sondern eher auf sprachliche Defizite des Autors zurückzuführen ist.

Insgesamt hat Frasers Zugriff aber nicht nur Nachteile. Dass er die Definition des Rechtsbegriffs umgeht, kann man ihm so sehr nicht übel nehmen. Er wirft mit diesem Ausweichmanöver einen gewaltigen Literaturballast über Bord, in dem es um Naturrecht und Positivismus und damit um die unendlichen rechtsphilosophischen Kernfragen des 20. Jahrhunderts geht. Die zu diesem Thema maßgeblichen Aussagen von Radbruch, Hart und vielen anderen Autoren wurden zu den Akten gelegt. Fraser zog es vor, zielgerichtet aus individuellen, philosophischen Vergangenheitsbetrachtungen auf aktuelle Themen zuzusteuern. Das hat auch etwas für sich und stiftet auf jeden Fall produktive Verwirrung. Es bleibt Frasers Verdienst, gezeigt zu haben, welche Katastrophen auch im heutigen juristischen Alltag Vorschriften oder Überzeugungen anrichten können, die für sich in Anspruch nehmen, Recht zu sein, und die nur aufgrund dieser Eigenschaft solche Wirkung entfalten, dass sie Widerspruch erschweren oder sogar als ausgeschlossen erscheinen lassen. Wer Recht hat, ist noch lange nicht sicher.

Thorsten Keiser 